\title{
Author Index for Volume 87
}

Abd Ellatif, M 1294

Abe T, 1153

Abeler, V 175

Aboagye, EO 783

Ackland, S 846

Adamson, KL 600

Addo S, 1354

Aft RL, 805

Agrez, MV 348

Aguirre, A 414

Agúndez, JAG 681

Ah-Weng, A 1386

Ahmad, SA 1182

Airoldi, L 1230

Akhmedkhanov, A 49

Akre, O 545

Al-Sakkaf, KA 909

Albanes, D 960

Alexander, FE 513

Allen, SG 1257

Alston, RD 1267

Altieri, A 1227

Amadori, D 868

Ameri, K 1173

Andachi, H 441

Andersen, MT 994

Andersen, SN 756

Anderson, A 1365

Anderson, TJ 334

Andreoni, M 1253

Andreyev, HJN 134

Arance, A 562

Arbea, L 158

Ardern-Jones, 905

Arends, MJ 1162

Arkenau, HT 702

Armstrong, S 268

Arndt, O 938

Arndt, V 519

Arslan, A 324, 1417

Ashcroft, L 562

Aso, T 377

Assadourian, S 1210

Ateenyi-Agaba, C 301

Atkin, SL 86

Atkinson, AD 381

Aumann, J 1328

Auvinen, A 511

Avizienyte, E 1128

Avril, A 615

Azli, N 1072

Babb, P 1267

Bachelot, T 1079

Baeten, CIM 344

Baeyens, A 1379

Bafaloukos, D 181

Baguley, BC 465

Bahlitzanakis, N 385

Bahlmann, F 635

Bailey, S 1092

Baillet, M 161

Baker, P 8

Balakrishnan, A 98
Balasubramanian, SP 1057

Ballini, J-P 1470

Ballmer-Hofer, K 106

Baracos, VE 1370

Barker, KT 446

Barnes, DM 208

Barnett, AA 246

Barraclough, R 423

Barratt, A 502

Barshishat, M 1314

Bartlett, JMS 654

Basso, O 524

Bateman, AC 834

Batley, SJ 1162

Batlle, A 471, 790

Baxter, K 733

Beare, S 15

Becker, JC 840

Becker, M 1328

Beckers, TL 924

Beex, LVAM 772

Begent, RHJ 600

Behr, Th 711

Beijnen, JH 608

Bekkers, RLM 373

Bell, BA 621

Bellocco, R 545

Benharroch, D 1314

Bennedsgaard, KM 194

Benner, A 1431

Benoy, I 1437

Beral, V 301

Beravs, K 1047

Berger, C 1197

Berghmans, T 694

Bermúdez Moretti, M 471

Bernard, JL 1197

Berney, DM 624

Bernstein, L 28

Bertuzzi, M 516, 956

Bevan, S 446, 1445

Bezemer, PD 75

Bhagavathula, N 457

Bianchi, S 65

Bidgood, C 733

Biglia, N 1287, 1294

Bijker, N 615

Birch JM 513, 1267

Biron, P 1079

Bishop, K 1445

Bisset, D 1072

Bissonnette, RP 555

Bjørge, L 1119

Bjørge, T 61

Björkstén, B 989

Blackhall, FH 381

Blackwell, RP 1257

Blagden, S 716

Blakey, D 600

Blant, SA 1470

Blay, JY 1079

Blesing, C 716

Boeing, H 519

Bokemeyer, C 729, 1066
Bonithon-Kopp, C 400

Bonneterre, J 1210

Boonstra, H 373

Borgoño, CA 1287

Bosetti, C 516, 956, 1227, 1230

Boshoff, C 301

Bouchier-Hayes, DJ 231

Boulamatsis, D 21

Boulay, J-L 630

Bourboulia, D 301

Bousarghin, L 301

Boutell, J 1162

Boutou, O 740

Bouvier, AM 400

Boven, E 665

Boyd, NF 876

Boyle, P 291

Brachetti, C 635

Bramhall, SR 161

Brant, R 1086

Bray, I 43

Breda, M 608

Brennan, P 43

Brenner, B 1404

Brenner, H 519

Bresnahan, B 1341

Bridge, JA 446

Brimmell, M 834

Brison, DR 381

Brock, C 1092

Bröcker, E-B 840

Brown, BL 909

Brown, NJ 1057

Brown, DJF 264

Brown, PD 161

Brown, SB 246

Brown, VL 208

Browne, J 476

Bruera, E 1370

Brunton, VG 1128

Brynes, RK 28

$\mathrm{Bu}, \mathrm{X} 28$

Bucana, CD 1182

Buccheri, G 1112

Buckels, JAC 161

Bullock, S 1445

Bundred, N 691

Burgers, JA 562

Burke, B 1173

Butow, PN 502

Bützow, R 1119

Byrne, MJ 491

Caca, K 689

Caine, GJ 1337

Caldas, C 1162

Califano, D 1479

Calvo, E 158

Cambeiro, M 158

Camero, A 129

Cameron, DA 1365

Campbell, NC 585

Cardar, PJ 687

Carpenter, L 301
Carrasco, R 432

Carroll, M 537

Cartwright, JE 673

Casabonne, D 301

Casas, A 471, 790

Cassidy, J 585

Cataliotti, L 615

Cella, D 1341

Cemazar, M 1047

Cerutti, J 1479

Chang, T-T 1449

Chang, Y-C 1449

Chapusot, C 400

Charlotte, F 1390

Charnley, RM 1034

Cheeseman, SL 393

Chen, C-J 966

Chen, HH-W 1449

Chen, R 1166

Chen, Y-F 1000

Chen, S-H 359

Chen, Y-L 359

Cherian, MG 1019

Chester, D 393

Chetty, U 8

Chhajlani, V 414

Chiarotti, L 1479

Ching, L-M 465

Chow, N-H 1449

Chow, W 281

Christiansen, M 994

Chung, H-K 405

Chung, J-H 405

Chung, SCS 91

Cini, G 497

Claes, K 1379

Clandinin, MT 1370

Clarke, PA 567

Clarke, SJ 277

Clausen, N 994

Cnattingius, S 974

Coer, A 1047

Colgan, T 763

Collaborative Group on Hormonal Factors in Breast Cancer, 1234

Collan, Y 1275

Colowick, AB 268

Colston, KW 555, 1340

Connolly, EM 231

Coppes, MJ 1086

Coradini, D 1105

Corfe, F 733

Correa García, S 471

Correale, P 720

Corrie, P 716

Cortes, J 158

Cosaert, J 825

Cotter, TG 1188

Coursaget, P 301

Coyle, P 533

Coze, C 1197

Craanen, ME 892

Craft, AW 1092 
Cresswell, J 1034

Crocetti, E 65

Crown, J 1365

Cruz, T 1253

Cugnenc, P-H 551

Cull, A 1099

Curtin, JF 1188

Cutress, RI 834

Dai, Y-C 1449

Daidone, MG 1105

Daigo, Y 1162

Dal Maso, L 1227

Dalgleish, AG 555, 1166

Damato, BE 1308

D'Ath, S 716

Davies, DC 621

Day, NE 1257

De Bruijn, P 144

de Graaf, M 659

de Jong, D 892

De Luca Cardillo, C 497

De Manzoni, G 171

de Mascarel, I 70

de Nigris, F 1479

De Paola, F 868

De Ridder, L 1379

Dearnaley, DP 905

DeCatris, M 15

Deichmann, M 1431

Delmotte, P 694

Delvaux, N 1

Dent, JT 393

Denton, AS 134

Di Leo, A 171

Diamandis, EP 339, 763, 1287, 1294

Diamandis, P 763

Diamond, EJ 726

Diaz-Gonzalez, JA 158

Dickinson, HO 746

Diep, A 294

Diep, CB 756

Dietz, A 938

Dillner, J 61, 175

Dirix, LY 1437

Distefano, MG 1145

Dixon, JM 8, 334, 950

Dobson, PRM 909

Domizio, P 779

Dommering, CJ 892

Done, K 733

Doni, L 497

Donskov, F 194

Dorahy, DJ 348

Doroshow, JH 281

dos Santos Silva, I 1195

Dosaka-Akita, H 751

Dredge, K 1166

Droz, JP 1079

Dubini, A 868

Dubus, L 1

Duffy, SW 65

Dumortier, A 1079

Dünne, A-A 711

Dunn, I 654

Durieux, J-F 1

Dyckhoff, G 938
Easton, DF 905

Eatough, JP 509

Ebert, MPA 91

Ecarnot-Laubriet, A 400

Edwards, SM 905

Eeles, RA 905, 1445

Efferth, T 251

Egeler, RM 1086

Egner, U 1431

Egrie, J 476

Ehlin, AGC 989

Ekbom, A 974, 989

Ekbom, A 545

Elliott, AM 585

Ellis, LM 1182

Elomaa, I 591

Endo, T 918

Engeland, A 61

Epoetin Alfa Study Group, 1341

Erlandsson, G 974

Ernst, E 479

Escobar, A 414

Esumi, H 898

Evans, TRJ 1365

Evans, S 567

Faivre, J 400

Falk, RT 54

Fallowfield, L 854, 1341

Fan, F 1182

Fan, LZ 1019

Fargeot, P 1210

Farvacques, C 1

Fears, TR 54

Fedier, A 1027

Fenig, E 1404

Fentiman, IS 615

Fernandez, L 1253

Ferrandina, G 1145

Ferrigno, D 1112

Fiaschi, AI 720

Fichtner, I 1328

Field, CJ 1370

Field, JK 1308

Fielding, D 491

Fielding, R 982

Fink, D 1027

Finlayson, CJ 446

Fioretto, LM 497

Fishell, E 876

Fisher, C 446

Fisker, R 194

Flanagan, AM 446

Fodstad, Ø 1281

Foekens, JA 772

Foley, D 231

Folz, BJ 711

Foo, W 982

Forbes, A 134

Forbes, K 733

Forman, S 281

Forrest, LM 264

Forsea, A-M 366

Forster, T 8

Försti, A 1162

Forsyth, A 654

Fouret, P 1390
Fracchioli, S 763

Fraier, D 608

Frame, MC 1128

Franceschi, S 314, 324, 516, 1227, 1230, 1253, 1417

Francini, G 720

Francis, RJ 600

Frangia, K 181

Frankel, P 281

Frappaz, D 1197

Frassineti, GL 868

French, JJ 1034

Frerichs, J 113

Freund, M 1341

Friery, OP 1339

Fritz, G 635

Froudarakis, M 385

Frye, RA 1479

Fujimori, T 1042

Fujino, Y 37

Fujisawa, T 81

Fujita, Y 1153

Fukuda, H 258, 790

Fukunaga, A 1140

Fukuoka, M 258

Fulignati, C 497

Funada, Y 1136

Furuhata, S 1454

Fusco, A 1479

Fusenig, NE 1301

Gaff, C 502

Gaffney, J 624

Gagnon, D 1341

Gallagher, CJ 850

Gallagher, R 1339

Gallus, S 516, 956, 1227

Gambi, A 868

Game, PA 533

Gao, DL 977

Garcia-Foncillas, J 158

García-Gamito, FJ 681

García-Martín, E 681

Garzon, C 158

Gazdar, AF 432

Geilen, CC 366

George, WD 1246

Gerritsen, WR 659

Gessi, M 1145

Geurti-Lackner, B 1445

Geurts-Moespot, J 772

Ghali, L 319

Giardina, G 763

Gil-Bazo, I 158

Gille, JJP 892

Giorgi, D 65

Giridharan, P 98

Gishizki, M 645

Gislefoss, RE 61

Gisselsson, D 202

Gius, D 805

Glaholm, J 938

Glanzmann, TM 1470

Glaspy, JA 268

Gnekow, A 945

Go, MYY 91

Goff, LK 779

Goff, L 537
Gogas, H 181

Gomez, F 1079

Gorzegno, G 1341

Goto, K 1360

Granato, AM 868

Grazia Porro, M 608

Greco, A 645

Green, AJ 600

Gregor, M 702

Greschniok, A 702

Grieve, RJ 1365

Griffioen, AW 344

Griffiths, K 1246

Griffiths, J 246

Grigor, KM 654

Grossbard, ML 372

Groves, F 511

$\mathrm{Gu}, \mathrm{X} 348$

Guastalla, JP 1079, 1210

Guizard, A-V 740

Gumbrell, L 600

Gurpide, A 158

Gyselman, VG 537

Hagiwara, A 1153

Hague, A 834

Haisma, HJ 659

Hakulinen, J 1119

Hall, CN 168

Hall, GH 86

Hall, K 846

Haller, U 1027

Hamburger, AW 1396

Hamilton, AS 294

Hamilton-Dutoit, S 1422

Hammel, P 551

Hammond, G 876

Hanks, GW 733

Hanselaar, AGJM 373

Harada, M 796, 1006

Harmey, JH 231

Harris, AL 1173

Harris, J 567

Harrison, KL 168

Hart, AAM 615

Hart, IR 859

Hartmann, JT 1066

Hartschuh, W 1301, 1431

Harwood, C 319

Hasan, T 1321

Hashimoto, D 1454

Hasle, H 524

Hass, HG 702

Hata, H 81

Hawkins, RA 8

Hayakawa, N 309

Hayase, H 1454

Hayashizaki, Y 1153

Hebert, C 1396

Hedley, AJ 982

Heighway, J 1308

Helboe, L 86

Helmerhorst, TJM 75

Henshaw, DL 1336

Hentrich, M 729

Herrera, I 339

Herrmann, R 630

Hicks, DJ 352 
Hida, N 796

Hill, E 168

Hillen, HFP 344

Hilson, AJ 600

Hino, S 1042

Hiraoka, K 1140

Hirst, DG 1339

Hitomi, Y 898

Hjalgrim, H 994

Hjalgrim, LL 994

Ho, C-K 529

Ho, C-L 1449

Ho, S-M 1411

Hochhauser, D 600, 1216

Hodgetts, 562

Hoefnagel, CA 705

Hoffman, BR 763

Hofmann, U 151

Hogenraad, R 1

Hogervorst, FBL 892

Hohenberger, P 478

Hoijer, M 113

Hokland, M 194

Hokland, P 994

Hoogsteen, IJ 665

Hoover, RN 54

Hope-Stone, LD 600

Horiot, J-C 478

Horne, G 381

Horsman, MR 1422

Hoshiyama, Y 309

Hosoda, A 441

Houlston, RS 446, 905, 1445

Hövelmann, S 924

Hoylaerts, M 1437

HPV Study Group, 324, 1417

Hsieh M-C, 1000

Hsu, H-K 529

$\mathrm{Hu}, \mathrm{J} 218$

Huget, Ph 1437

Hughes, CM 1339

Hulme, MJ 8

Hummel, M 1462

Hunt L, 1204

Hutchinson, OC 783

Huzarski, T 888

Hwang, J-H 187

Ichikawa, Y 1153

Ikarashi, Y 1454

Ikeguchi, M 883

Imamura, K 898

Ioannovich, J 181

Ishikawa, T 1153

Iskander, L 1287

Ito, $\mathrm{H} 441$

Ito, M 1006

Itoh, K 796, 1006

Itoh, T 751, 1140

Ivanusa, T 1047

Iyer, NG 1162

Izumi, H 751

Jack, WJL 8

Jackson, RA 905

Jackson, S 319

Jacobs, IJ 537

Jadeja, JS 268
Jaffe, H 301

Jager, D 113

Jakubowska, A 888

Jalava, P 1275

James, R 1216

Jamieson, GG 533

Jansen, S 608

Japan Collaborative Cohort Study Group, 309

Jarva, H 1119

Jellum, E 61, 175

Jenkins, V 854

Jensen, JJ 194

Jiang, C-C 218

Jin, C 202

Jin, Y 202

Jobo, T 81

Joel, SP 393

Joensuu, H 591

Jonat, W 1480

Jones, A 1365

Jones, RJ 1128

Jong, R 876

Jonson, T 202

Jordan, S 624

Jordan, VC 449

Jørgensen, P 994

Julien, J-P 615

Jung, EG 151

Junghahn, I 1328

Junnikkala, S 1119

Justice, G 268

Kaibara, N 441, 883

Kaina, B 635

Kaiser, SA 449

Kakinuma, R 1360

Kamata, Y 81

Kameya, T 81

Kämpgen, E 840

Kamura, T 796, 1006

Kanai, T 81

Kanelopoulos, P 21

Kanz, L 729, 1066

Kao, E-L 529

Karthikeyan, NP 98

Katagiri, K 796

Kato, K 898, 1140

Katoh, H 751, 1140

Katsaros, D 763

Kawaguchi, K 441

Kawaguchi, T 309

Kawakami, N 31

Kawamata, H 1042

Kawasaki, H 441

Kay, E 231

Kaye, SB 813, 1072

Kefala, G 181

Kehoe, ST 1337

Kehri, W 938

Kelleher, DK 1462

Kelsell, DP 208

Kelsey, AM 1267

Kennedy, ICS 1365

Kessler, J 268

Kestell, P 465

Kiely, MA 1099

Kiessling, R 414
Kikuchi, R 1136

Kikuchi, S 309

Kilkarni, R 1092

Killen, ME 8

Kim, C-W 405

Kim, D-J 405

Kim, I-J 187

Kim, JI 314

Kim, JY 314

Kim, S-W 187

Kim, W-H 187

Kingston, JE 779

Kinoshita, I 751

Kirby, JA 1034

Kirk, J 502

Kishimoto, Y 441

Kitamura, A 441

Klaproth, H 729

Kleeman, H-W 238

Klemenz, R 106

Kliakhandler, IL 449

Klump, B 702

Knap, MM 1422

Koda, M 441

Koenig, KL 49

Koetsier, M 113

Kogut, N 281

Kohlhagen, G 665

Kohno, K 751

Kollmannsberger, C 729

Kolonel, LN 54

Kondo, S 1140

Kondo, T 309

Kong, X 519

Koomägi, R 251

Korenblit, C 414

Koskela, P 61

Kosmas, C 21

Kostourou, V 673

Kotnik, V 1047

Koukourakis, GV 385

Koukourakis, MI 385

Kouzarides, T 1162

Kreienberg, R 519

Krishna, S 621

Kronqvist, P 1275

Kroon, BBR 705

Kruyt, FA 659

Krzic, M 1047

$\mathrm{Ku}, \mathrm{J}-\mathrm{L} 187$

Kubin, T 729

Kubota, K 1360

Kubota, T 377

Kuczyk, M 729

Kudoh, S 258

Kuehl, J 945

Kuopio, T 1275

Kuramoto, H 81

Kurihara, H 171

Kyrgias, G 385

La Vecchia, C 291, 516, 956, 1230, 1272

Lacau-St-Guily, J 1390

Laffer, U 630

Lafitte, J-J 694

Lagiou, P 956

Lagrange, M 630
Lai, W-W 1449

Laight, A 1354

Lam, T-H 982

Lambe, M 974

Lansdown, MRJ 687

Larcombe, I 1204

Larsson, O 414

Latif, Z 654

Lau, EMC 982

Lau, B 218

Laurent Puig, P 400, 551

Lauriola, L 1145

Lavery, KM 938

Le Vay, JH 1365

Leach, L 854

Leavitt, RD 938

Lecher, B 1462

Lecoeur, C 537

Ledermann, JA 1216

Lee, DH 314

Lee, DS 314

Lee, J-M 529

Lee, REJ 1092

Lee, W-Y 1449

Lee, Y-C 529

Lees, NP 168

Legge, F 1145

Lehrer, S 726

Lehtinen, M 61, 175

Lence, J 1253

Leonard, P 1216

Leonard, RCF 1365

Leong, L 281

Leung, GM 982

Leung, WK 91

Levenson, AS 449

Levi, F 291, 516, 1227, 1230

Levi, I 1314

Levi, J 846

Lévy, P 551

Lewis, C 846

Lewis, CE 1173

Leygue, E 1411

Lian, S-T 1000

Lie, AK 175

Lieb, W 840

Lieberman, BA 381

Lieverst, J 608

Lillington, DM 779

Lin, S-R 1000

Lip, GYH 1337

Lippert, BM 711

Little, J 585

Littlewood, TJ 1341

Liu, D 423, 783

Liu, D 423

Lobb, EA 502

Lomax, L 562

Loos, WJ 144

López, M 414

Lothe, RA 756

Loukopoulos, D 181

Lowe, DG 537

Lowy, A 630

Løvig, T 756

Lu, Y-J 446

Lubiński, J 888

Lucchini, F 291 
Lundqvist, A 414

Luo, L-Y 339

Luostarinen, T 61, 175

Lurie, H 1404

Lynch, P 1099

Mælandsmo, GM 1281

Maaya, MB 381

Macdonald, CD 1166

MacGrogen, G 70

Mack, TM 294

Mackey, J 1370

Madsen, HO 994

Maeda, Y 796, 1006

Maggiano, N 1145

Magklara, A 1294

Maher, EJ 134

Maire, F 551

Maitra, A 432

Makino, M 441

Malfertheiner, P 91

Maliepaard, M 665

Malonne, H 475

Man, H-W 1166

Mandahl, N 202

Manders, P 772

Manganelli, A 720

Mansi, J 1365

Mansi, JL 1340

Manuelian, T 1119

Marchal, S 1

Marcussen, N 194, 1422

Margison, GP 168

Margolin, K 281

Marrelli, D 171

Marriott, JB 1166

Marsden, JR 1386

Marshall, JF 859

Marsili, S 720

Martínez, C 681

Martin, B 694

Martin, J 600

Martin, L 400

Martin, LJ 876

Martin-Algarra, S 158

Martins, C 202

Marty, C 106

Marx, G 846

Mascaux, C 694

Maslanyj, MP 1257

Massobrio, M 763

Massuger, LFAG 373

Mathew, G 533

Mathijssen, RHJ 144

Mathoulin-Pelissier, S 70

Matsumoto, T 1360

Matsuo, K 751

Mattern, J 251

Maune, S 938

Mayer, A 716

Mayer, F 729

Maytin, EV 1321

Mbidde, E 301

Mbulaiteye, S 301

McArdle, CS 264, 1246

McCarty, MF 1182

McElgunn, CJ 892

McGuire, S 1445
McIntyre, MA 8

McKeown, SR 1339

McMillan, DC 264

McNally, RJQ 513, 1267

McNamara, M 281

McNulty, P 1341

McWilliams, DF 567

Mee, TJ 1257

Meert, A-P 694

Meijer, CJLM 75, 324, 1417

Meijer, GA 892

Meijer, HP 113

Meiser, B 502

Meitz, JC 905

Melbye, M 994

Melchers, WJG 373

Meling, GI 756

Memari, N 1294

Menko, FH 892

Meri, S 1119

Mertens, F 202

Messiaen, L 1379

Messinese, S 720

Metzner, B 1066

Micard, S 551

Michaud, DS 960

Michon, J 1197

Mild, G 630

Miller, WR 950

Miller, WR 8, 334, 688

Milroy, R 264

Mini, E 497

Minkin, S 876

Minna, JD 432

Miranda, C 645

Mitchell, G 1445

Mitchell, P 491

Miura, Y 309

Miyamoto, M 1140

Mizoue, T 37, 309

Molano, M 324, 1417

Mölenkamp, G 945

Molina, A 281

Moll R, 711

Monnier, A 1210

Monnier, P 1470

Montgomery, SM 989

Moon, SE 457

Mooney, LM 909

Morelli, C 1479

Moretti, R 497

Morgagni, P 171

Morgan, H 733

Morgan Jr, R 281

Morita, T 1153

Moriyama, H 1136

Mork, J 61

Morris, TM 567

Mössner, J 689

Mott, M 1204

M'Rabet, L 113

Muller, GW 1166

Munoz, N 324, 1417

Murawaki, Y 441

Murkin, A 905

Murphy, LC 1411

Murray, MM 1339

Musk, AW 491
Myers, JC 533

Näher, H 1431

Nagata, C 31

Nakagawa, K 258

Nakakubo, Y 1140

Nakanishi, M 1153

Nakashiro, K 1042

Namer, M 1210

Nanni, O 868

Naredi, P 194

Navarro, V 158

Nederlof, PM 892

Negoro, S 258

Negri, E 291, 516, 956, 1227 , 1230

Nehls, O 702

Nej, K 888

Nemunaitis, J 161

Neri, B 497

Neri, D 106

Nesland, JM 1281

Neumann, C 840, 1328

Newsham, A 854

Newton, R 301

Ng, EKW 91

Ng, H-K 218

Ngoan, LT 37

Nicholls, K 854

Nicolas, AI 158

Nieweg, OE 705

Niho, S 1360

Nikitakis, NG 1396

Nishimura, M 751

Nishimura, Y 258

Nishiwaki, Y 1360

Nishizuka, I 1153

Nitsche, A 1328

Niu, Y 1411

Niu, J 348

Niya, F 796

Nobbenhuis, MAE 75

Noguchi, T 1136

Nomura, AMY 54

Nonaka, Y 1006

Nooter, K 144

Nowak, AK 491

Nunn, J 1308

Nyari, TA 746

O'Brien, MER 481

O'Byrene, KJ 15

O’Byrne, J 268

Odermatt, B 106

O'Donnell, D 1321

Oechsle, K 1066

Offersen, BV 1422

O'Grady, T 231

Ohmatsu, H 1360

Ohno, E 81

Ohno, Y 309

Okazaki, Y 1153

Oliver, RTD 624

Oliverio, G 868

Olsen, J 524

Olsen, JH 524

O'Malley, D 600

Omotehara, F 1042
Onadim Z, 779

O’Neill VJ 933

Oosterhoff, D 659

Orenberg, EK 938

Orfanos, CE 366

Orpana, A 591

Ortel, B 1321

Ortiz, RM 1253

Oshikiri, T 1140

Osman, S 783

Österlund, P 591

Otsuka, I 377

Overgaard, J 1422

Owens, DW 1128

Özdağ, H 1162

Paci, E 65

Packham, 834

Paesmans, M 694

Pallisgaard, N 994

Pals, G 892

Panagiotou, P 181

Pang, JC-s 218

Pantalone, D 497

Papadakis, ED 1013

Papadopoulos, MC 621

Paraskeva, C 352

Parikh, AA 1182

Park, J-G 187

Park, Y-H 187

Parker, L 746

Parkin, DM 301

Parmar MKB 815

Pasini, F 171

Patterson, LH 1339

Pauson, AW 1246

Pease, KM 449

Pectasides, D 181

Pedersen, KB 1281

Pellizzaro, C 1105

Pellizzoni, C 608

Pepper, MS 537

Pereira, P 225

Perol, D 1197

Perotti, C 790

Perotti, C 471

Perren, TJ 1365

Perumal, K 98

Peters, TJ 733

Peterse, JL 705

Peterse, JL 615

Petersson, M 414

Petrioli R, 720

Pettersson, F 555

Philcox, JC 533

Philip, I 1079

Philip, T 1197

Piard, F 400

Picot, V 70

Pierotti, MA 645

Pietinen, P 960

Pike, MC 54

Pilz, L 1301

Pinedo, HM 659, 665

Pitchforth, E 1221

Pizarro, RM 681

Plagens, A 212

Playle, LC 352 
Pluzanska, A 938

Polymeris, M-E 1287

Polyzos, A 181

Pommier, Y 665

Ponder, BAJ 1162

Ponnelle, T 400

Ponzone, R 1287, 1294

Poon, W-S 218

Poppe, B 1379

Porschen, R 702

Posso, H 324, 1417

Pottier, D 740

Povey, AC 168

Pozzessere, D 720

Pratt, VC 1370

Prescott, RJ 8

Press, MF 294

Price, E 779

Price, L 1092

Price, P 716, 783

Proby, CM 208

Pukkala, E 61

Puopolo, M 763

Qian, W 815

Qin, Q 977

Qualtrough, D 352

Quattrone, A 497

Quinn, MJ 1267

Quoix, E 825

Radford, JA 381

Rageot, D 400

Raisova, M 366

Rakowsky, E 1404

Ramaswamy, A 711

Ramazeilles, C 1072

Rana, B 574

Ranelletti, FO 1145

Ranzi, V 645

Rappaport, EB 294

Raschko, J 281

Rating, D 945

Ray, RM 977

Ray-Coquard, I 1079

Razavi, D 1

Rebattu, P 1079

Reed, MWR 1057

Reeves, G 301

Reinmuth, N 1182

Repo, H 591

Retalis, G 385

Reuter, J 630

Reynolds, MA 1396

Rezza, G 1253

Rhim, J-H 405

Ribecco, AS 497

Richards, D 268

Richards, FJ 393

Richiardi, L 545

Richmond, I 86

Rick, O 72

Riebeling, C 366

Rigault de la Longrais, IA 763

Ritchie, LD 585

Rittgen, W 151

Riva, A 1072

Rivory, LP 277
Roagna, R 1287

Robbins, A 600

Robbins, M 733

Roberts, A 1092

Roberts, DJH 246

Robinson, BWS 491

Robinson, SP 673

Roccato, E 645

Roché, H 1072, 1210

Roche-Nagle, G 231

Rochlitz, C 630

Rodriguez, J 158

Rofe, AM 533

Rognum, TO 756

Rohloff, J 689

Romanidis, K 385

Romundstad, PR 289, 971

Ronderos, M 324, 1417

Rosing, H 608

Rosselli del Turco, M 65

Rossi, G 268

Roviello, F 171

Rozen, R 225

Rozendaal, L 75

Rudland, PS 423

Ruefenacht, UB 1027

Russell, E 1221

Russell, ST 580

Ruszniewski, P 551

Rutgers, EJTh 615, 705

Ryan, A 537

Ryan, G 491

Ryder, LP 994

Ryder, WDJ 381

Ryu, Y-S 405

Saadoun, S 621

Sabatino, M 720

Sabbaghian, N 225

Sacca, P 790

Sahmoud, T 950

Sahmoud, T 334

Sajedi, M 1086

Sakakura, C 1153

Sakata, K 309

Salazar-Onfray, F 414

Salgado, R 1437

Salutari, V 1145

Salvestrini, F 720

Sandercock, J 815

Sanders, DSA 1386

Santella, RM 966

Santelli, G 1479

Saragoni, L 171

Sarmati, L 1253

Sasaki, T 918

Sato, $\mathrm{H} 81$

Sato, Y 796

Sauk, JJ 1396

Saunders, PTK 688

Sayer, HG 729

Scambia, G 1145

Scarpi, E 868

Scatizzi, M 497

Schöffski, P 1066

Schadendorf, D 151, 840

Scheffer, GL 665

Schellens, JHM 608
Scheper, RJ 665

Schievella, AR 225

Schlüper, HMM 665

Schleicher, J 1066

Schleucher, N 1066

Schmidt, K 479

Schmidt, M 635, 924

Schmiegelow, K 994

Schoemaker, NE 608

Schofield, J 246

Scholes, AGM 1308

Schoppmeyer, K 689

Schott, H 106

Schouten, JP 892

Schulz, J 161

Schwartz, B 1314

Schwartzberg, L 268

Schwarz, VA 1027

Schwendener, RA 106

Scorilas, A 763, 1287, 1294

Scott, HR 264

Scott, R 348

Scott, RJ 888

Sculier, J-P 694

Segal, A 491

Seifart, C 212

Seifart, U 212

Sekhon, J 225

Selby, PJ 1099

Sena, L 600

Senaratne, SG 1340

Sentjurc, M 1047

Serraino, D 1253

Serrano, A 414

Sersa, G 1047

Seymour, K 1034

Seymour, MT 393, 1216

Shahbakhti, H 600

Shamash, J 850

Shamash, J 624

Sharlin, D 1321

Sharma, RA 15

Sharma, SK 600

Sharp, D 733

Sharp, L 585

Shaw, J 716

Shepherd, JH 537

Shi, W 119

Shibata, S 281

Shichijo, S 1006

Shichinohe, T 1140

Shields, F 15

Shimada, H 1153

Shimizu, A 1454

Shimizu, H 31

Shimomura, K 1153

Shin, S-J 1000

Shin, HR 314

Shinohara, T 1140

Shiota, G 441

Shipley, J 446

Shirakawa, K 1454

Shore, RE 49

Siavash, H 1396

Sibson, DR 423

Siemann, DW 119

Sigstad, E 175

Simpson, HW 1246
Simpson, J 1257

Simpson, JF 281

Sinha, AK 1321

Sinha, D 511

Siris, N 1404

Sismondi, P 1287

Sismondi, P 1294

Skillen, AW 1092

Skinner, J 1257

Skinner, R 1092

Skjærven, R 289, 971

Slama, R 740

Slater, S 850

Slaviero, KA 277

Slevin, ML 850

Sloane, JP 1055

Smeets, RL 113

Smith, A 567

Smith, SL 1308

Snell, L 1411

Sodha, N 1445

Soler-Michel, P 1079

Somasundaram, ST 98

Somlo, E 281

Somlo, G 281

Sone, T 659

Song, DH 574

Soosaipillai, A 339

Souhami, RL 478

Soulitzis, N 1013

Spandidos, DA 1013

Sparén, P 989

Sparreboom, A 144

Speight, PM 859

Speirs, V 687

Spinelli, R 608

Spira, A 740

Spott, C 729

Springer, C 600

Stagger, S 726

Steinmann, A 840

Steward, WP 15

Stewart, ME 938

Stirling, D 1166

Stock, RG 726

Stoeltzing, O 1182

Stone, J 876

Stone, NN 726

Storey, A 319

Stratton, MR 905

Strieth, S 1301

Stuart, M 334, 950

Stürmer, T 519

Suh, K-S 187

Suh, Y 405

Sulkes, A 1404

Sun, C-A 966

Sung, JJY 91

Suthers, GK 502

Suzuoki, M 1140

Svoboda, KM 449

Swart, M 608

Swartz, HM 1047

Sweep, CGJ 772

Sykes, J 733

Szedlak, M 151

Takada, K 127 
Takagi, T 1153

Takahashi, M 796

Takahashi, S 1153

Takatsuka, N 31

Takeda, K 258

Takeno, S 1136

Talamini, R 516, 1227, 1230

Tamakoshi, A 309

Tanaka, M 258

Tang, M 432

Tanis, PJ 705

Tannapfel, A 689

Tannock, I 478

Tannock, IF 238

Tavani, A 956

Taylor, PR 960

Taylor, R 1445

Tchekmedyian, NS 268

Temam, S 1390

ten Bokkel Huinink, WW 608

Terada, M 1454

Terheyden, P 840

Terracciano, L 630

Terret, C 1072

Tesdale, AL 8

Thach, TQ 982

Thatcher, N 562

The Cancer Research UK/BPG UK Familial Prostate Cancer Study Collaborators, 905

Thews, O 1462

Thierens, H 1379

Thomas, AL 15

Thomas, DB 977

Thomas, GJ 859

Thome, M 1431

Thoresen, S 175

Thoresen, SØ 61

Thorstensen, L 756

Tisdale, MJ 580

Tjan-Heijnen, VCG 772

Todo, S 796

Tokui, N 37, 309

Tong, CY-k 218

Toniolo, P 49

Tookman, A 1480

Torri, V 815

Touzé, A 301

Townsend, PA 834

Toy, E 1365

Toyoshima, H 309

Trichopoulos, D 289, 956, 971

Troncoso, P 432

Tsavaris, N 21

Tsiompanou, E 600

Tsoutsos, D 181

Tsuda, N 1006
Tucker, K 502

Tunstall, RG 246

Turci, D 868

Turkes, A 1246

Turnbull, LW 86

Turrini, M 497

Twelves, CJ 933, 1072

Uchida, D 1042

Uchida, Y 1136

Uganda Kaposi's Sarcoma Study Group, 301

Underwood, MA 654

United Kingdom Childhood Cancer Study Investigators, 1257

Vaccarella, S 1253

Vadiaka, M 21

Valdés Olmos, RA 705

van Beusechem, VW 659

Van Bockstaele, D 1437

van den Bergh, H 1470

van den Brule, AJC 75, 324 1417

van der Meulen, IH 659

Van der Pol, M 1221

van Ham, MAPC 373

van Hattum, AH 665

van Kesteren, C 608

Van Marck, E 1437

van Schooten, RJ 892

Van Tienoven, TH 772

Van Trappen, PO 537

van't Land, B 113

Varani, J 457

Vasey, PA 1072

Vatten, LJ 971

Vatten, LJ 289

Vaupel, P 1462

Velmurugan, R 98

Veneroni, S 1105

Ventura, L 1105

Verdebout, J-M 694

Verhoeven, ICL 344

Verlato, G 171

Vermeulen, P 1437

Vernillet, L 1072

Vernon, DI 246

Verweij, J 144

Vezzosi, V 65

Viglietto, G 1479

Virtamo, J 960

Virtanen, M 960

Vishwakarma, RA 98

Voitot, H 551

Volm, M 251

Volpi, A 868
Von der Maase, H 194

Von Wichart, P 212

Voorhorst, FJ 75

Vral, A 1379

Wabinga, H 301

Wagner, S 840

Wagnières, G 1470

Wagstaff, 344

Wakasugi, H 1454

Wang, J-Y 1000

Wang-Gohrke, S 519

Wang, HB 91

Wang, J-Y 359

Wang, L-Y 966

Wang, R 446

Wang, Y-T 529

Wämberg, F 691

Ward III, JE 449

Warmuth-Metz, M 945

Warner, N 716

Watanabe, I 1454

Watanabe, S 1370

Watanabe, J 81

Waters, JS 481

Waters, R 1386

Watson, PH 1411

Watson, SA 567

Watters, AD 654

Webley, S 600

Weiderpass, E 324, 1417

Welslau, M 729

Werner, JA 711, 938

West, DW 54

Westphal, S 945

Weytjens, R 1437

Whitley, GStJ 673

Wiegant, J 202

Wijnen, JTh 892

Williams, RD 1445

Williams, S 905

Williamson, R 491

Wilson, P 850

Wilson, G 393

Winther, JF 524

Wistuba, II 432

Wittekind, C 689

Wittenberg, G 840

Witzigmann, H 689

Wolf, M 212

Wolfe, MM 574

Wolff, JEA 1086

Wolff, JEA 945

Wong, C-M 982

Wong, WK 1034

Wong, P 238

Workman, P 783

Wright, EP 1099
$\mathrm{Wu}, \mathrm{AH} 54$

$\mathrm{Wu}, \mathrm{C}-\mathrm{H} 1000$

Wu, D-C 529

Wu, D-M 966

Wu, M-T 529

Wyke, AW 1128

Yaffe, M 876

Yamada, A 1006

Yamada, M 258

Yamada, Y 751

Yamagishi, H 1153

Yamamoto, N 258

Yamana, H 796

Yamazaki, K 751, 918

Yamori, T 918

Yang, B-C 359

Yang, J-Y 187

Yao, M 574

Yashima, K 441

Yasuoka, R 1153

Yates, RA 1354

Yatsuya, H 309

Ye, Z 977

Yeh, C-S 1000

Yin, X-I 218

Yip, PSF 982

Yoo, KY 314

Yoon, K-A 187

Yoon, Y-B 187

Yoshida, T 1454

Yoshimura, T 309

Yoshimura, T 37

You, S-L 966

Young, BD 537, 779

Young, MA 502

Yousef, GM 1287, 1294

$\mathrm{Yu}, \mathrm{C} 202$

$\mathrm{Yu}, \mathrm{J} 91$

Zagari, M 1341

Zannoni, GF 1145

Zappa, M 65

Zeleniuch-Jacquotte, A 49

Zhang, FW 805

Zhao, L 465

Zhou, L-F 218

Zhu, JL 524

Ziegler, J 301

Ziegler, RG 54

Zinke, J 689

Zipfel, PF 1119

Zocchi, G 497

Zoli, W 868

Zucker, JM 1197 